\title{
BMJ Open Diverse perspectives on hospitalisation events among people with dementia: protocol for a multisite qualitative study
}

\author{
Andrea Gilmore-Bykovskyi (D) , ${ }^{1,2}$ Quinton Cotton, ${ }^{1}$ Jennifer Morgan, ${ }^{1}$ Laura Block ${ }^{1}$
}

To cite: Gilmore-Bykovskyi A, Cotton Q, Morgan J, et al. Diverse perspectives on hospitalisation events among people with dementia: protocol for a multisite qualitative study. BMJ Open 2021;11:e043016. doi:10.1136/ bmjopen-2020-043016

- Prepublication history for this paper is available online. To view these files, please visit the journal online (http://dx.doi. org/10.1136/bmjopen-2020043016).

Received 21 July 2020 Revised 25 November 2020 Accepted 25 January 2021

A) Check for updates

(c) Author(s) (or their employer(s)) 2021. Re-use permitted under CC BY-NC. No commercial re-use. See rights and permissions. Published by BMJ.

${ }^{1}$ Nursing, University of Wisconsin-Madison, Madison, Wisconsin, USA

${ }^{2}$ Division of Geriatrics, Department of Medicine, University of Wisconsin-Madison School of Medicine \& Public Health, Madison, Wisconsin, USA

Correspondence to

Dr Andrea Gilmore-Bykovskyi; algilmore@wisc.edu

\section{ABSTRACT}

Introduction People living with dementia (PLWD) are more likely to experience hospitalisation events (hospitalisation, rehospitalisation) than those without dementia. Many hospitalisation events, particularly rehospitalisation within 30 days of discharge, are thought to be avoidable. Yet our understanding of dementiaspecific risk and protective factors surrounding avoidable hospitalisation is limited to specific intersetting transitions and predominantly clinician perspectives. Broader insights are needed to design accessible and effective solutions for reducing avoidable hospitalisations. We have designed the Stakeholders Understanding of Prevention Protection and Opportunities to Reduce HospiTalizations (SUPPORT) Study to address these gaps. The objectives of the SUPPORT Study are to elicit and examine family caregiver, community and hospital providers' perspectives on avoidable hospitalisation events among PLWD, and to identify opportunities for effective prevention.

Methods and analysis We will conduct a multisite, descriptive qualitative study to interview around 100 family caregivers, community and hospital providers. We will identify and sample from regions and communities with higher socio-contextual disadvantage and hospital utilisation, and will aim to recruit individuals representing diverse racial/ethnic backgrounds. Interviews will follow a descriptive qualitative design in conjunction with constant comparison techniques to sample divergent situations and events. We will employ a range of analytical approaches to address specific research questions including thematic (inductive and deductive), comparative and dimensional analysis. Interviews will be conducted individually or in focus groups and follow a semistructured interview guide. Ethics and dissemination The study is approved by the University of Wisconsin-Madison Institutional Review Board. Informed consent procedures will incorporate steps to evaluate capacity to provide informed consent in the event that participants express concerns with thinking or memory or demonstrate challenges recalling study details during the consent process to ensure capacity to consent to participation. A series of publicly available reports, seminars and symposia will be undertaken in collaboration with collaborating organisation partners.

\section{INTRODUCTION}

Dementia is a progressive, irreversible neurodegenerative condition that leads to pervasive losses in cognitive abilities and functioning. ${ }^{1}$
Strengths and limitations of this study

By sampling diverse groups of stakeholders, we aim to include a broad range of under-represented perspectives, contexts, and situations to inform our understanding of risk and protective factors surrounding hospitalisation events among people living with dementia.

- The study methods will generate a large amount of rich qualitative data, which will provide insights into how hospitalisation events are perceived by various stakeholders.

- The study recruitment plan aims to include family caregivers, in particular, who may appraise and experience hospitalisation and rehospitalisation differently than providers.

- A limitation of the study methods is that data will not be collected directly from individuals living with dementia, but from other informants supporting care who may have distinct perspectives.

People living with dementia (PLWD) often rely heavily on both formal health and social services, as well as informal neighbourhood and community-level resources to meet their basic needs. ${ }^{2}{ }^{3}$ Unpaid family caregivers are often at the centre of care management for PLWD and provide the vast majority of direct care while also navigating health and service access and utilisation. ${ }^{4-6}$ However, considerable evidence indicates that the availability, accessibility, and scope of care management and supportive services for PLWD are insufficient to fully address the range and complexity of dementia-related care needs. ${ }^{7-11}$ Inadequate management and supports for PLWD, and their caregivers, have been cited as major contributor to frequent, avoidable transitions into and out of hospital settings. ${ }^{12} 13$

In addition to exposing PLWD to additional risk for negative outcomes such as falls, delirium and accelerated cognitive decline, hospitalisation also contributes to significant distress among PLWD, as the consistent environments, caregivers and routines they rely on are disrupted during hospital stays. ${ }^{14-17}$ In 
addition to being hospitalised at disproportionately high rates, PLWD are also more likely to experience rehospitalisation, defined as readmission to the hospital setting within 30 days of discharge. ${ }^{18-20} \mathrm{~A}$ considerable proportion of hospitalisation events are considered avoidable, yet effective solutions to preventing hospitalisation events for PLWD remain elusive. ${ }^{18} 2122$ Many interventions designed to reduce avoidable hospitalisation events only target the immediate post-discharge period or rehospitalisation, and thus may not be effective in reducing initial entry into hospital settings from the community. ${ }^{13}$ Furthermore, PLWD have been excluded from the development and testing of many of these interventions, thus the utility of these approaches for reducing rehospitalisation risk among PLWD is unknown. ${ }^{22-25}$

In response to these challenges, health system and policy reform in many countries, including the USA, France and England, has targeted rehospitalisation events due to the excessive and preventable nature of adverse outcomes and high financial costs associated with these admissions. ${ }^{23}{ }^{24}$ In the USA, rehospitalisation rates for many conditions appear to be higher than other countries despite sweeping policy changes enacted with the 2009 Affordable Care Act to incentivise improved care coordination and reduce rehospitalisation. ${ }^{23-25}$ A major focus of this legislation was the value-based Hospital Readmissions Reduction Program, which levies financial penalties against hospitals that have higher than national average rehospitalisation rates. ${ }^{26}$ Emerging evidence suggests that hospitals serving predominantly disadvantaged populations have borne the brunt of the financial rehospitalisation penalties, leading to cuts in 'non-essential' services, such as caregiver support programmes, that could serve to benefit PLWD and their caregivers. ${ }^{27}{ }^{28}$ Because people from historically disadvantaged or underserved backgrounds are both more likely to develop dementia and to reside in disadvantaged service areas with fewer supportive resources, rehospitalisation policies may exacerbate existing disparities in dementia care and utilisation experienced by those communities. ${ }^{29}{ }^{30}$ Experiences with available community supports, hospitalisation and posthospitalisation care may also vary based on individual and geographical characteristics of the service areas in which patients reside (eg, resource-rich or resourcerestricted neighbourhoods). Thus, it is vital that efforts to identify potential contributory and preventive factors surrounding hospitalisation events among PLWD incorporate perspectives of populations and regions at greatest risk for being underserved.

Despite growing evidence that policy efforts to improve transitional care have not resulted in desired improvements in hospitalisation use among PLWD, ${ }^{13}{ }^{22-25}$ our understanding of potentially modifiable risk and protective factors specific to this population remains limited. An informant-centred approach to elicit, examine and compare the perspectives of diverse stakeholders with firsthand experience is needed to develop a comprehensive understanding of important risks and unmet needs that contribute to hospitalisation events, as well as existing protective factors that should be maintained. Additionally, garnering input from family caregivers as well as community and hospital providers can aid in the identification of discrepant perspectives around the need for hospitalisation or utility of various supports for PLWD and their caregivers.

To achieve these goals, we designed the Stakeholders Understanding of Prevention Protection and Opportunities to Reduce HospiTalizations (SUPPORT) Study. The SUPPORT Study aims to elicit and examine family caregiver, community and hospital providers' experiences with and perspectives regarding avoidable hospitalisation events. The SUPPORT Study will address the following primary research aims:

1. To explore risk and protective factors surrounding hospitalisation events from the perspective of diverse stakeholders from under-resourced and higher risk environments.

2. To identify conditions and events that precipitate hospitalisation events and perceived preventability of precipitating factors.

3. To compare various stakeholder viewpoints regarding causes, preventability and consequences of hospitalisation events for PLWD.

4. To examine perceived accessibility and quality of supportive services and resources that could reduce hospitalisation risk.

5. To identify sources of common barriers and facilitators to utilisation of beneficial supportive services and resources that could reduce hospitalisation risk.

A secondary aim of the study is to facilitate and enable comparisons, specifically (1) between various types of resources, supports, and risks factors and hospitalisation risk, and (2) between different stakeholder perspectives surrounding hospitalisation events.

\section{METHODS AND ANALYSIS}

\section{Study design}

A qualitative descriptive design will be used to collect data from family caregivers, community and hospital providers using semistructured interviews. Qualitative description is well suited to the study's overarching goals of examining and comparing perspectives around hospitalisation events among PLWD and summarising key findings in relation to specific research questions. ${ }^{31-33}$ To ensure emergent conceptual understandings of participant experiences are grounded in the data, interviewers will employ constant comparison techniques throughout interviews by intentionally sampling divergent situations and events with the goal of lending greater clarity and ongoing clarification of identified concepts through theoretical sampling of additional cases and events. Arising from social interactionism paradigmatic methodologies, such as Grounded Theory and Grounded Dimensional Analysis (GDA), constant comparison techniques involve both sampling (data collection) and analytical procedures 
designed to facilitate discovery of variations in relationships between concepts and associated dimensions and contextual factors identified from interview data. ${ }^{345}$ The constant comparison technique is often used to facilitate theoretical sampling or refinement of emerging theoretical categories identified in interviews and data analysis. ${ }^{36}$

\section{Study settings and participants}

To obtain a rich variety of perspectives from various caregiving, community and hospital contexts, a tiered sampling strategy will be used to first purposely identify regions where populations have higher socio-contextual and hospital utilisation disadvantage, and second to identify specific points of contact within these regions to facilitate study recruitment with the goal of maximising opportunities for representativeness in the overall sample.

\section{Tier 1}

Purposive sampling will be used to identify three to four areas from different regions across the USA that have higher than national-average neighbourhood level socioeconomic disadvantage (ie, socio-contextual disadvantage) and regional rehospitalisation rates, and that represent communities with diversity in racial and ethnic minority populations. This sampling approach is intended to: (1) yield insights into experiences around hospitalisation events within communities that are exposed to higher levels of socio-contextual disadvantage, (2) provide a rich opportunity for evaluating hospitalisation events within regions where rehospitalisation events are more common and unintended consequences of monetary disincentives may be most evident, and (3) facilitate recruitment within diverse racial/ethnic populations that are often underrepresented in dementia research.

Several approaches will be used to facilitate tier 1 purposive sampling. First, the Area Deprivation Index (ADI), a validated measure of neighborhood-level sociocontextual disadvantage, will be used to identify regions with higher levels of socioeconomic disadvantage. ${ }^{37}$ The ADI incorporates 17 poverty, education, housing and employment indicators derived at the census block group level, and provides a percentile ranking of the 'level of disadvantage,' with 1 indicating lowest levels of disadvantage and 100 indicating highest levels of disadvantage. ${ }^{37}$ Specifically, this study will use the ADI to identify communities within the $20 \%$ most disadvantaged in the USA from different regions and higher than national average rehospitalisation rates. To determine the latter, Medicare claims data from years 2013 to 2014 will be used. Lastly, we will review regional census data to evaluate and balance representation of populations from diverse racial and ethnic composition in selecting regions.

\section{Tier 2}

To facilitate recruitment of potential participants, we will implement multifaceted recruitment strategies for each region by establishing points of contact within relevant coalitional networks and community health, social service and hospital-based organisations. Example organisations include those serving older adults or focusing on Alzheimer's disease and related dementias, such as senior centres, Area Agencies on Aging and local Alzheimer's Association chapters. The recruitment strategy will be informed through key informant interviews with representatives from organisations identified through both existing relationships and web searching. Key informant interviews will also guide identification of other local points of contact to facilitate recruitment. We will document contacts made and referral numbers by recruitment source.

Convenience and purposive sampling will be used to identify potential participants with relevant experiences to inform study questions. Individual participant recruitment will be accomplished through a combination of in-person, telephone and email contact via established points of contact which will help facilitate dissemination of study recruitment materials including flyers and invitation letters for potential participants. Interested participants will contact the researchers directly either by telephone, email or in person during one of the visits that will be made by researchers to each region. Visits to each region will serve to establish relationships with key stakeholders, garner a more in-depth understanding of regional and community-specific challenges, and facilitate in-person data collection and reports of research findings to the local communities.

All eligibility screening will take place with participants during initial contact. Specifically, eligibility criteria for family caregivers include: (1) currently providing or having provided unpaid care to a person with Alzheimer's disease or related dementias at least monthly, and (2) ability to complete interviews in English. Eligibility criteria for community and hospital providers include: (1) current or previous experience providing care to people with Alzheimer's disease or related dementias, and (2) ability to complete interviews in English. The interviewers will engage in theoretical sampling to guide further questions regarding contextual factors, events, decisionmaking and consequences associated with hospitalisation events as informed by emergent analysis. As family caregiver perspectives, particularly from under-represented populations, are under-represented in currently available literature on hospitalisation events, we will aim to oversample from this participant group. Across selected study site regions, we will recruit and interview approximately 100 participants, including 70 family caregiver participants, 15 community providers and 15 hospital providers. We anticipate that this number of participants will be required to achieve data saturation across research questions, given potential differences in experience by study site and participant group. For example, experiences around rehospitalisation may differ between urban and rural contexts; therefore, sufficient interviews by context will be required to achieve data saturation. Anticipated sample size and recruitment strategies may be modified if theoretical sampling reveals additional heterogeneity 
in core findings and concepts that merit more extensive sampling or if more interviews are needed within certain participant groups to enable adequate comparisons in perspectives of hospitalisation events.

\section{Participant involvement}

Throughout study execution, patient and public involvement will be incorporated through regular consultation with a community advisory board with diverse membership representing patient and caregiver perspectives. Consultation with the community advisory board, specifically the Community Advisors on Research Design and Strategies local to Madison, Wisconsin, will focus on feedback and guidance on recruitment materials, data collection procedures including interview questions, and garnering input on interpretation of study findings. To date, the community advisory board has been instrumental in advising recruitment approach and reviewing recruitment and data collection materials. Similar advice will be sought from community advisory boards local to the selected study sites. Community and health system organisations and the general public will also be involved in dissemination of information through invitations to review and provide comments and questions in response to a non-peer-reviewed report. The final report will be distributed to participants and community stakeholders on the conclusion of the study.

\section{Data collection}

Data collection for this study will take place over 24-30 months as based on time needed to establish points of contact across selected regions and to recruit and interview participants. A semistructured interview guide will be used to guide data collection during interviews and focus groups based on overall study objectives (box 1).

Generally, interviews will begin with more broad, openended questions and become more focused as each interview and the overall study progresses. Data collection will take place over the phone or in a private room at a location that is convenient to the participant (such as a private room in a public library). While focus groups will only be conducted in person, individual interviews may take place in person or via phone. Decisions regarding individual interview or focus group format will be based on the research team's need for data reflecting individual versus group perspectives, respectively, along with participant preferences and convenience and logistics/ feasibility of different data collection options. In some situations, we anticipate that some participants may have a preference toward either individual interviews or focus groups. All participants will be provided the option to opt into a different format of participation wherever feasible. In light of logistical considerations with travel to various regions and time constraints caregivers face, we anticipate that most participants will complete an individual interview via phone.

Individual interviews are expected to last approximately $60-90 \mathrm{~min}$ in duration with focus groups lasting

\section{Box 1 Sample interview questions*}

\section{Family caregivers}

What does day-to-day caregiving look and feel like for you?

The following prompts may be used to elicit more input, if needed:

a. What do you like best (what are the positive things) about being a caregiver or providing care?

b. What do you feel are some of the biggest challenges you face as a caregiver?

c. Are there specific experiences as a caregiver that you feel are more stressful and need your immediate attention? Give me a few examples.

d. What kind of support or resources would make your life easier as a caregiver? (This could be something to help you or help the person you are caring for.)

e. How do you balance your caregiving responsibilities with the rest of your life?

f. What things are keeping the person you care for as healthy and out of the hospital?

- Can you briefly describe a time when you OR the person you care for experienced something urgent or unexpected that required immediate attention or action?

a. Briefly describe times when the person you care for has been to the emergency room, urgent care or hospital.

b. Can you talk about the events leading up to their hospital stay?

c. What kinds of things went well during this period of time? What didn't go well?

d. What kinds of things did you need help with then? Did you get that help? Who gave it to you? How did it go? Example prompt: Is there anything you didn't get help with that would have made your life easier?

e. Are there any kinds of help that would've helped keep the person you care for at home?

f. Has the person you care for gone to the hospital two or more times in 1 month? What happened?

\section{Community providers}

- Can you share a bit about how you interact with people with dementia and their caregivers in your current role?

- What are the biggest needs or concerns for people in your community experiencing changes in memory and their caregivers?

a. Are there gaps in care or services that may be impacting hospital use?

b. Which gaps in care and services are not well addressed? What might be getting in the way?

c. If you could change one thing to better meet the needs of persons with dementia and their caregivers, what would it be?

- Can you talk about a time that you interacted with a person with dementia or their caregiver before or after a decision to go to the hospital?

Hospital providers

- In your experience, what does care for people with dementia who enter the hospital look like?

- Are there ways these hospitalisations could be prevented?

- What types of services might better help people with dementia and their caregivers to avoid unnecessary hospitalisation?

*These sample interview questions are not exhaustive but are representative of types of questions that will be asked of each participant type.

approximately 90-120 min in duration, with consideration for caregiver time constraints. Exact interview duration will vary based on participant availability and the 
Table 1 Interview guidance

\begin{tabular}{ll}
\hline $\begin{array}{l}\text { Interviewing } \\
\text { strategy }\end{array}$ & Specific guidance \\
\hline Building rapport & $\begin{array}{l}\text { Don't jump into the } \\
\text { questions right away; } \\
\text { how the participant is } \\
\text { doing and engage with } \\
\text { answer }\end{array}$ \\
\hline $\begin{array}{l}\text { Practising } \\
\text { therapeutic } \\
\text { communication }\end{array}$ & $\begin{array}{l}\text { Validate participant } \\
\text { experiences }\end{array}$ \\
\hline
\end{tabular}

Grant permission to speak 'We've heard of all kinds of about difficult subjects without being judged

\section{exp} study so far. Throughout our interview today, keep in mind that no matter how you perceive your experiences, we want to hear them. You are the expert here, and we're going to work together to get as much out of our time together as we can.'

Reflect participant feelings 'It sounds like you are and language feeling very angry about what happened. Is that right?'

Normalise experiences 'Research suggests that and emotions

'Research suggests that
this experience is not uncommon.'

Draw from literature to help normalise participant experiences, reactions and emotions.

\begin{tabular}{|c|c|c|}
\hline \multirow[t]{2}{*}{$\begin{array}{l}\text { Moving the } \\
\text { interview along }\end{array}$} & $\begin{array}{l}\text { Acknowledge information } \\
\text { and emotions }\end{array}$ & $\begin{array}{l}\text { 'I can really hear how much } \\
\text { this situation affected you.' }\end{array}$ \\
\hline & Use transition techniques & $\begin{array}{l}\text { 'Thank you for all that } \\
\text { you're sharing! Let me take } \\
\text { a moment to look at the } \\
\text { next questions.' }\end{array}$ \\
\hline \multirow[t]{7}{*}{$\begin{array}{l}\text { Probing for } \\
\text { information }\end{array}$} & \multirow[t]{2}{*}{ Ask for more details } & $\begin{array}{l}\text { 'Could you tell me more } \\
\text { about the situation?' }\end{array}$ \\
\hline & & $\begin{array}{l}\text { 'Could you give a concrete } \\
\text { or specific example?' }\end{array}$ \\
\hline & $\begin{array}{l}\text { Restate and/or circle back } \\
\text { to questions }\end{array}$ & $\begin{array}{l}\text { Restate the question(s) } \\
\text { in a different way. Use } \\
\text { phrases such as 'I want } \\
\text { to circle back to what you } \\
\text { said about (prior question). } \\
\text { Could you tell me more } \\
\text { about the situation?' }\end{array}$ \\
\hline & $\begin{array}{l}\text { Ask participants for their } \\
\text { opinions about the cause } \\
\text { of something }\end{array}$ & $\begin{array}{l}\text { 'Why do you think that } \\
\text { happened?' }\end{array}$ \\
\hline & $\begin{array}{l}\text { Ask participants how a } \\
\text { situation felt }\end{array}$ & $\begin{array}{l}\text { 'How did you feel during } \\
\text { that situation? Was there } \\
\text { anything that you felt could } \\
\text { have made it better?' }\end{array}$ \\
\hline & \multirow[t]{2}{*}{ Use open questions } & $\begin{array}{l}\text { 'How do you feel about the } \\
\text { situation?' }\end{array}$ \\
\hline & & $\begin{array}{l}\text { 'What are some things that } \\
\text { trouble you most?' }\end{array}$ \\
\hline
\end{tabular}

extent of information and experiences they have to share. At the end of each interview, participants will be asked to fill out a short demographic survey and for caregivers, a questionnaire on self-reported level of stress.
During each interview, the interviewer will maintain detailed memos through notes taken about participant responses to capture the interviewer's reflections regarding emergent concepts and their relationships. Interviewers will also note interview question wording or interview strategies that appeared to be challenging for participants, or other relevant observations. Memos will be referred to in later interpretative stages of analysis or may be used to inform additional theoretical sampling and questioning in future interviews. ${ }^{38}$ Throughout data collection and analysis, as facilitated through memoing, we will identify initial interviews where emergent concepts, categories and themes may need to be clarified or deepened. Participants who have contributed an initial interview that has been identified for follow-up will be invited to participate in a second supplemental interview. Participants will be given the opportunity to consent (or not) to recontact during the verbal informed consent process.

As caregiver participants may be under a significant amount of stress related to caregiving responsibilities, interviewers will engage various strategies in order to ensure participant comfort and build rapport during the interview (table 1).

These strategies highlight the use of therapeutic communication and open-ended questions to encourage participants without leading them to particular answers, periodic reminders that questions are optional and that the interview may be paused or stopped at any time to mitigate potential emotional distress during interviews and provision of tailored resource lists. Additionally, interviewers will be trained to offer caregiver participants a list of tailored, local resources to be created and provided immediately after the interview. All participants will receive a $\$ 35$ honorarium on completion of each interview.

All interviews will be audio recorded, transcribed verbatim and de-identified. De-identification will involve removal of identifiable personal information in addition to the names of specific individuals or settings (eg, clinic names) and will be double checked by a second reviewer prior to using transcripts in analysis. Transcribed interviews will also be checked against the audio recording for accuracy and edited as needed to reflect the original recording.

\section{Data analysis}

De-identified interview transcripts will be analysed using NVivo qualitative data analysis software V.12. A range of analytical approaches will be used to answer specific study questions, including inductive and deductive thematic, comparative and dimensional analysis. ${ }^{34-36} 39$ Provided the breadth of domains and experiences that will be elicited in interview questions, it is anticipated that analytical approach will be specific to the different research questions, using methodological approaches that are most appropriate to the complexity, variation and depth of resultant data. For example, thematic analysis may be 
applied to questions where determining similarities across interviews is useful, such as characterising perceived accessibility and quality of resources used to support PLWD that may reduce hospitalisation events. ${ }^{39}$ GDA techniques may be best suited to analyse data regarding decision-making and processes surrounding rehospitalisation events. ${ }^{34}$ A variant of Grounded Theory, GDA is designed to enable an understanding of social processes (rehospitalisation) and the conditions and consequences that characterise variations in these events, as well as how participants understand and make sense of these events so as not to impose a researcher-generated hypothesis. ${ }^{34}$ Lastly, comparative analysis may be well suited to aims focused on evaluating differences in cases and appraisal of contextual factors presented by different participant types—or for evaluating different types of hospitalisation events. $^{39}$

Consistent with established procedures to achieve theoretical sampling, preliminary data analysis will be conducted concurrently with data collection. ${ }^{40}$ Iterative and ongoing analysis will help shape needed revisions in interview approach and inform areas to focus or extend future interviews. In particular, we will aim to continually identify opportunities to sample divergent situations and events (ie, constant comparison approach). ${ }^{36}$ In some situations, ongoing analysis will inform our understanding of participant perspectives that may be lacking or would be informative to study aims. In general, situations and events will constitute the unit of analysis, and coding will progress through sequential review of transcripts by multiple coders. Examples of potential situations and events that will be sampled include: decisions to use or not use certain supportive resource under differing sets of circumstances, experiences with discharge instructions and related actions taken, and interpretation and decisions made in response to observed changes in behaviour or health preceding hospitalisation events. Across all analysis approaches, we will use transcripts to complete analysis and will engage multiple researchers in the analysis process to enhance trustworthiness and rigour. Where possible, member checking will also take place to receive feedback on the interpretation of study findings. Where appropriate to the methodology, coding schemas will be developed and iteratively refined and intercoder agreement will be evaluated in application of the schemas to interview data. All substantive decisions will be documented in internal standard operating procedures.

\section{ETHICS AND DISSEMINATION}

\section{Ethical considerations}

This study was reviewed and approved by a Human Subjects Institutional Review Board (IRB) at the University of Wisconsin-Madison. The study was approved with a waiver of written consent to reduce the risk for loss of confidentiality. Study participants will be provided with an information sheet that reviews study risks and benefits, and provide verbal informed consent. Informed consent procedures will incorporate steps to evaluate capacity to provide informed consent in the event that participants express concerns with thinking or memory or demonstrate challenges recalling study details during the consent process to ensure capacity to consent to participation.

\section{Dissemination of findings}

We will use a variety of methods to ensure our work will be properly disseminated to academic, clinical and public audiences. The publication of our study protocol provides an important step in the dissemination of our work. We have sought to provide a detailed description of the methodology we will employ to collect the perspectives of stakeholders from multiple sites in the USA. Study findings continue to be disseminated in high-quality, peer-reviewed journals and presented in key national and international conferences. Additionally, we will work to create a non-peer-reviewed report on our findings that will be shared with collaborating organisations and participants as a way to disseminate our findings to a broader audience. The researchers will aim to make de-identified interview data available through IRB-approved procedures and on establishment of data sharing agreements.

\section{DISCUSSION}

Few studies have evaluated and compared multiple stakeholder perspectives on hospitalisation events among PLWD, particularly among high-risk and underrepresented communities. This study will enable a broader understanding of hospitalisation events through systematic exploration of diverse stakeholder perspectives by identifying potential protective and risk factors, and evaluating perceived relevance and utility of various preventive resources and supports. By focusing broadly on experiences and views surrounding hospitalisation events and garnering input from different stakeholders, it may be possible to examine differences and similarities between different types of hospitalisation events and different perspectives. This may shed light on varying interpretations of precipitating events surrounding hospitalisation, which may reveal previously undefined sources of miscommunication or misaligned decision-making that could exacerbate avoidable hospitalisations among PLWD.

When examining communication and decision-making around hospitalisation and rehospitalisation events, it may be possible that the nature of formal versus family caregiving may play a role. For example, multidisciplinary clinicians identify opportunity for improved intersetting communication, care management and care planning to prevent rehospitalisation. ${ }^{4-43}$ Interestingly, clinicians also cite that patients and caregivers may have unrealistic expectations for goals of care in post-acute care settings, indicating the need for greater collaboration between clinicians and families. ${ }^{4344}$ In contrast, family caregivers are often the only consistent link with firsthand knowledge of care received across various points in the care continuum and may view their role during transitions 
of care as one of advocacy. ${ }^{45}$ Caregivers may identify issues common during handoffs or transitions that other providers would not have had the opportunity to observe or recognise as problematic, or to witness relevant implications and consequences of actions and inactions surrounding transitions. In fact, a systematic review found that when family caregivers are integrated into the discharge process, a $25 \%$ reduction in rehospitalisation can be achieved, signalling their critical role in transitions of care. ${ }^{47}$ Caregivers may also advocate for the inclusion of the PLWD's perspective, which research has demonstrated is often left out during decision-making and care planning. ${ }^{48} 49$ The current study holds promise in contributing to the emerging discussion on provider and family caregiver perspectives on communication and decision-making during transitions, and whether there are concordant or diverging views on roles and boundaries.

Generating more insights from the perspective of family caregivers in particular is a major priority of this study as the lack of knowledge about caregiver perspectives makes it challenging if not impossible to effectively target resources, design interventions, and shape policies in accordance to their real and perceived needs and limitations. Of note, the present study will aim to understand what caregivers perceive as sources of protection and resilience. ${ }^{50}$ This deviates from many dementia care studies which focus predominantly on deficits. ${ }^{50}$ Understanding sources of innate protection and strength within caregiving networks will enable discovery of ways in which certain policies or interventions could inadvertently disrupt or minimise the benefits of these sources of protection. Insights of community and hospital providers will also present important opportunities to understand policy consequences—or potential improvements in relevant policy - that caregivers may be less familiar with.

Existing evidence that can help inform approaches to reduce avoidable hospitalisation events among PLWD focuses on a narrow subset of intersetting transitions or only on rehospitalisation events, which may inadvertently overlook opportunities to assemble a more comprehensive picture of structural supports and systems of broad relevance to PLWD and their caregivers. This study may illuminate best practices along with areas for improvement in the integration of community, post-acute and acute care services to improve care continuity for PLWD. Practices around inclusion of caregivers in hospital admission and discharge may vary due in part to policy differences such as the Caregiver Advise, Record, Enable Act which requires hospitals to provide family caregivers with information on provision of needed care for care recipients on discharge. ${ }^{51}$

Finally, this study also presents a concerted effort to elicit perspectives from socio-contextually disadvantaged groups, diverse populations and largely under-represented communities that may be disproportionately impacted by unintended consequences of value-based payment policies, and that may face additional specific barriers to addressing the needs of PLWD. By focusing data collection on specific areas that experience higher levels of socioeconomic disadvantage and rehospitalisation and represent diverse racial and ethnic populations, we will be able to develop an understanding of hospitalisation events specific to vulnerable and marginalised communities that remain under-represented in dementia research. ${ }^{52}$

A potential limitation of this study is the fact that we are not integrated into all study sites. Although we plan to travel to each study site and will use local study partners to facilitate recruitment, engagement and response rates to invitations to participate across geographical regions may vary. Additionally, longitudinal engagement of local study partners necessitates intentional planning, time, resources and follow-up, which can pose some challenges. Another limitation of the present study is that individuals with dementia will not be included due to the additional burden posed by phone-based capacity assessment and interviews. While centring the perspectives of dementia caregivers will provide important insights into challenges faced by PLWD, we recognise that PLWD may contribute important otherwise inaccessible insights into what hospitalisation means for them and ways they could be better supported.

\section{Twitter Quinton Cotton @QuintonCotton}

Acknowledgements The authors would like to thank Dr Amy Kind and Barbara Bowers for providing expert guidance and review throughout study stages. The authors would also like to thank Clark Benson for support in manuscript formatting.

Contributors $A G-B, Q C$ and $L B$ contributed to the conceptualisation and design of this study. AG-B, JM, QC and $L B$ contributed to the design and drafting of the study protocol. AG-B, JM, QC and LB will contribute to data collection. AG-B, JM, QC and $\mathrm{LB}$ contributed to the manuscript, and reviewed and approved the final version of the paper.

Funding This work is supported by the National Institute for Minority Health and Health Disparities of the National Institutes of Health under award number R01MD010243 (PI Kind) and the National Institute of General Medical Sciences of the National Institutes of Health under award number R25GM083252 (PI Carnes). Dr Kind and AG-B also receive funding from the National Institute on Aging under award numbers P30AG062715 (PI Asthana) and K76AG060005 (PI AG-B).

Disclaimer The content is solely the responsibility of the authors and does not necessarily represent the official views of the National Institutes of Health.

Competing interests None declared.

Patient and public involvement Patients and/or the public were involved in the design, or conduct, or reporting, or dissemination plans of this research. Refer to the Methods section for further details.

Patient consent for publication Not required.

Provenance and peer review Not commissioned; externally peer reviewed.

Open access This is an open access article distributed in accordance with the Creative Commons Attribution Non Commercial (CC BY-NC 4.0) license, which permits others to distribute, remix, adapt, build upon this work non-commercially, and license their derivative works on different terms, provided the original work is properly cited, appropriate credit is given, any changes made indicated, and the use is non-commercial. See: http://creativecommons.org/licenses/by-nc/4.0/.

\section{ORCID iD}

Andrea Gilmore-Bykovskyi http://orcid.org/0000-0003-4930-3558

\section{REFERENCES}

1 McKhann GM, Knopman DS, Chertkow H, et al. The diagnosis of dementia due to Alzheimer's disease: recommendations from the National Institute on Aging-Alzheimer's Association workgroups on diagnostic guidelines for Alzheimer's disease. Alzheimers Dement 2011;7:263-9.

2 Bökberg C, Ahlström G, Karlsson S. Utilisation of formal and informal care and services at home among persons with dementia: a crosssectional study. Scand J Caring Sci 2018;32:843-51. 
3 Brodaty H, Donkin M. Family caregivers of people with dementia. Dialogues Clin Neurosci 2009;11:217-28.

4 Papastavrou E, Kalokerinou A, Papacostas SS, et al. Caring for a relative with dementia: family caregiver burden. $J$ Adv Nurs 2007;58:446-57.

5 Cohen LW, Zimmerman S, Reed D, et al. Dementia in relation to family caregiver involvement and burden in long-term care. J Appl Gerontol 2014;33:522-40.

6 Ponnala S, Block L, Lingg AJ, et al. Conceptualizing caregiving activities for persons with dementia (PwD) through a patient work lens. Appl Ergon 2020;85:103070.

$7 \mathrm{Li} \mathrm{H}$. Unmet service needs: a comparison between dementia and non-dementia caregivers. Home Health Care Serv Q 2012;31:41-59.

8 McCabe M, You E, Tatangelo G. Hearing their voice: a systematic review of dementia family caregivers' needs. Gerontologist 2016;56:e70-88.

9 Peterson $\mathrm{K}$, Hahn $\mathrm{H}$, Lee AJ, et al. In the information age, do dementia caregivers get the information they need? Semi-structured interviews to determine informal caregivers' education needs, barriers, and preferences. BMC Geriatr 2016;16:164.

10 Werner NE, Stanislawski B, Marx KA, et al. Getting what they need when they need it. identifying barriers to information needs of family caregivers to manage dementia-related behavioral symptoms. Appl Clin Inform 2017;8:191-205.

11 Phillipson L, Jones SC, Magee C. A review of the factors associated with the non-use of respite services by carers of people with dementia: implications for policy and practice. Health Soc Care Community 2014;22:1-12.

12 Fortinsky RH, Downs M. Optimizing person-centered transitions in the dementia journey: a comparison of national dementia strategies. Health Aff 2014;33:566-73.

13 Hirschman KB, Hodgson NA. Evidence-Based interventions for transitions in care for individuals living with dementia. Gerontologist 2018;58:S129-40.

14 Bail K, Goss J, Draper B, et al. The cost of hospital-acquired complications for older people with and without dementia; a retrospective cohort study. BMC Health Serv Res 2015;15:91.

15 Fogg C, Griffiths P, Meredith P, et al. Hospital outcomes of older people with cognitive impairment: an integrative review. Int J Geriatr Psychiatry 2018;33:1177-97.

16 Fong TG, Jones RN, Marcantonio ER, et al. Adverse outcomes after hospitalization and delirium in persons with Alzheimer disease. Ann Intern Med 2012;156:848-56.

17 Reilly JC, Houghton C. The experiences and perceptions of care in acute settings for patients living with dementia: a qualitative evidence synthesis. Int J Nurs Stud 2019;96:82-90.

18 Phelan EA, Borson S, Grothaus L, et al. Association of incident dementia with hospitalizations. JAMA 2012;307:165-72.

19 Callahan CM, Arling G, Tu W, et al. Transitions in care for older adults with and without dementia. J Am Geriatr Soc 2012;60:813-20.

20 Fillenbaum G, Heyman A, Peterson BL, et al. Use and cost of hospitalization of patients with AD by stage and living arrangement: CERAD XXI. Neurology 2001;56:201-6.

21 Desai U, Kirson NY, Ye W, et al. Trends in health service use and potentially avoidable hospitalizations before Alzheimer's disease diagnosis: a matched, retrospective study of US Medicare beneficiaries. Alzheimers Dement 2019;11:125-35.

22 Piraino E, Heckman G, Glenny C, et al. Transitional care programs: who is left behind? A systematic review. Int $J$ Integr Care 2012;12:e132.

23 Gusmano M, Rodwin V, Weisz D, et al. Comparison of rehospitalization rates in France and the United States. $J$ Health Serv Res Policy 2015;20:18-25.

24 Kristensen SR, Bech M, Quentin W. A roadmap for comparing readmission policies with application to Denmark, England, Germany and the United States. Health Policy 2015;119:264-73.

25 Kociol RD, Lopes RD, Clare R, et al. International variation in and factors associated with hospital readmission after myocardial infarction. JAMA 2012;307:66-74.

26 Hansen LO, Young RS, Hinami K, et al. Interventions to reduce 30-day rehospitalization: a systematic review. Ann Intern Med 2011:155:520-8.

27 Gu Q, Koenig L, Faerberg J, et al. The Medicare Hospital readmissions reduction program: potential unintended consequences for hospitals serving vulnerable populations. Health Serv Res 2014;49:818-37.
28 Gilman M, Adams EK, Hockenberry JM, et al. California safety-net hospitals likely to be penalized by ACA value, readmission, and meaningful-use programs. Health Aff 2014;33:1314-22.

29 Zeki Al Hazzouri A, Haan MN, Osypuk T, et al. Neighborhood socioeconomic context and cognitive decline among older Mexican Americans: results from the Sacramento area Latino study on aging. Am J Epidemiol 2011:174:423-31.

30 Yaffe K, Falvey C, Harris TB, et al. Effect of socioeconomic disparities on incidence of dementia among biracial older adults: prospective study. BMJ 2013;347:f7051.

31 Neergaard MA, Olesen F, Andersen RS, et al. Qualitative description - the poor cousin of health research? BMC Med Res Methodol 2009;9:52.

32 Bradshaw C, Atkinson S, Doody O. Employing a qualitative description approach in health care research. Glob Qual Nurs Res 2017;4:233339361774228

$33 \mathrm{Kim} \mathrm{H}$, Sefcik JS, Bradway C. Characteristics of qualitative descriptive studies: a systematic review. Res Nurs Health 2017;40:23-42.

34 Kools S, McCarthy M, Durham R, et al. Dimensional analysis: broadening the conception of grounded theory. Qual Health Res 1996;6:312-30.

35 Glaser BG, Strauss AL. Discovery of grounded theory: strategies for qualitative research. Routledge, 2017.

36 Charmaz K. Grounded theory: methodology and theory construction. In: Wright JD, ed. International encyclopedia of the social \& behavioral sciences. 2nd edn. Oxford: Elsevier, 2015: 402-7.

37 Kind AJH, Buckingham WR. Making neighborhood-disadvantage metrics accessible - The Neighborhood Atlas. N Engl J Med 2018;378:2456-8.

38 Birks M, Chapman Y, Francis K. Memoing in qualitative research: probing data and processes. J Res Nurs 2008;13:68-75.

39 Braun V, Clarke V. Using thematic analysis in psychology. Qual Res Psychol 2006;3:77-101.

40 Corbin J, Strauss A. Techniques and procedures for developing Grounded theory. In: Basics of qualitative research. 3rd edn. Thousand Oaks: SAGE Publications, Inc, 2008. https://methods. sagepub.com/book/basics-of-qualitative-research

41 Vasilevskis EE, Ouslander JG, Mixon AS, et al. Potentially avoidable readmissions of patients discharged to post-acute care: perspectives of hospital and skilled nursing facility staff. J Am Geriatr Soc 2017:65:269-76.

42 Clark B, Baron K, Tynan-McKiernan K, et al. Perspectives of clinicians at skilled nursing facilities on 30-day hospital readmissions: a qualitative study. J Hosp Med 2017;12:632-8.

43 Feder SL, Britton MC, Chaudhry SI. They need to have an understanding of why they're coming here and what the outcomes might be. Clinician perspectives on goals of care for patients discharged from hospitals to skilled nursing facilities. $J$ Pain Symptom Manage 2018:55:930-7.

44 Jacobsen JML, Schnelle JF, Saraf AA, et al. Preventability of hospital readmissions from skilled nursing facilities: a consumer perspective. Gerontologist 2017;57:1123-32.

45 Sellars M, Chung O, Nolte L, et al. Perspectives of people with dementia and carers on advance care planning and end-of-life care: a systematic review and thematic synthesis of qualitative studies. Palliat Med 2019;33:274-90.

46 Gillick MR. The critical role of caregivers in achieving patientcentered care. JAMA 2013;310:575-6.

47 Rodakowski J, Rocco PB, Ortiz M, et al. Caregiver integration during discharge planning for older adults to reduce resource use: a metaanalysis. J Am Geriatr Soc 2017;65:1748-55.

48 Donnelly S, Begley E, O'Brien M. How are people with dementia involved in care-planning and decision-making? an Irish social work perspective. Dementia 2019;18:2985-3003.

49 Pecanac KE, Wyman M, Kind AJH, et al. Treatment decision making involving patients with dementia in acute care: a scoping review. Patient Educ Couns 2018;101:1884-91.

50 O'Dwyer ST, Moyle W, Taylor T, et al. In their own words: how family carers of people with dementia understand resilience. Behav Sci 2017;7 doi: $10.3390 /$ bs7030057

51 Caregiver Advise, Record, and Enable (CARE) act, STAT. S676B. 2015 https://legislation.nysenate.gov/pdf/bills/2015/S676B

52 Gilmore-Bykovskyi AL, Jin Y, Gleason C, et al. Recruitment and retention of underrepresented populations in Alzheimer's disease research: a systematic review. Alzheimers Dement 2019;5:751-70. 